\title{
Optimizing acute stroke care organization: a simulation study to identify gains and obstacles to overcome
}

\author{
M.M.H.Lahr', D.J. Van der Zee², G.J.Luijckx³, E.Buskens ${ }^{1,2}$
}

1 University of Groningen, University Medical Center Groningen, Health Technology Assessment, Department of Epidemiology, Groningen, Netherlands.

2University of Groningen, Department of Operations, Faculty of Economics \& Business, Groningen, Netherlands.

${ }^{3}$ University of Groningen, University Medical Center Groningen, Department of Neurology, Groningen, Netherlands.

\section{BACKGROUND}

- Treatment with intravenous thrombolysis (IVT) is substantially underused.

- Previous studies have demonstrated clear benefits in terms of time saved or identified process indicators for separate elements of stroke pathway delivery. ${ }^{1,2}$

- Nevertheless a broader overview of pathway set-up is lacking and current interventions target isolated elements of the stroke pathway.

\section{OBJECTIVE}

To estimate the cumulative potential for improving IVT utilization by implementing best practices on the organization of the stroke pathway.

\section{METHOD}

- Ischemic stroke patients were prospectively ascertained in four hospitals from February to August 2010, as part of a centralized organizational model. ${ }^{3}$

- Simulation modeling was used to compare the performance of the current pathway, best practices based on literature review, and an optimized model.

- The interventions investigated included efforts aimed at patient response and mode of referral, prehospital triage and treatment, and intra-hospital delays.

\section{OUTCOME MEASURES}

- The primary outcome measure was thrombolysis utilization.

- Secondary measures were onset-treatment time (OTT) and the proportion of patients with excellent functional outcome (mRS 0-1) at 90 days.

\section{RESULTS}

- Of 280 patients with ischemic stroke, 125 (44.6\%) arrived at the hospital within 4.5 hours, and $61(21.8 \%)$ received IVT.

- The largest improvements in outcome measures can be expected when:

Patient response is limited to 15 minutes (IVT rate $+5.8 \%$; OTT -6 minutes; excellent $\mathrm{mRS}$ scores $+0.2 \%$ ).

Door-to-needle time is reduced to 20 minutes (IVT rate $+4.8 \%$; OTT -28 minutes; excellent mRS scores $+3.2 \%$ ).

911 calls are increased to $60 \%$ (IVT rate $+2.9 \%$; OTT -2 minutes; excellent mRS scores +0.2\%).

- The combined implementation of all potential best practices could increase IVT rates by $19.7 \%$ and reduce OTT by 56 minutes.

Figure 1. Acute stroke pathway: description of activities.

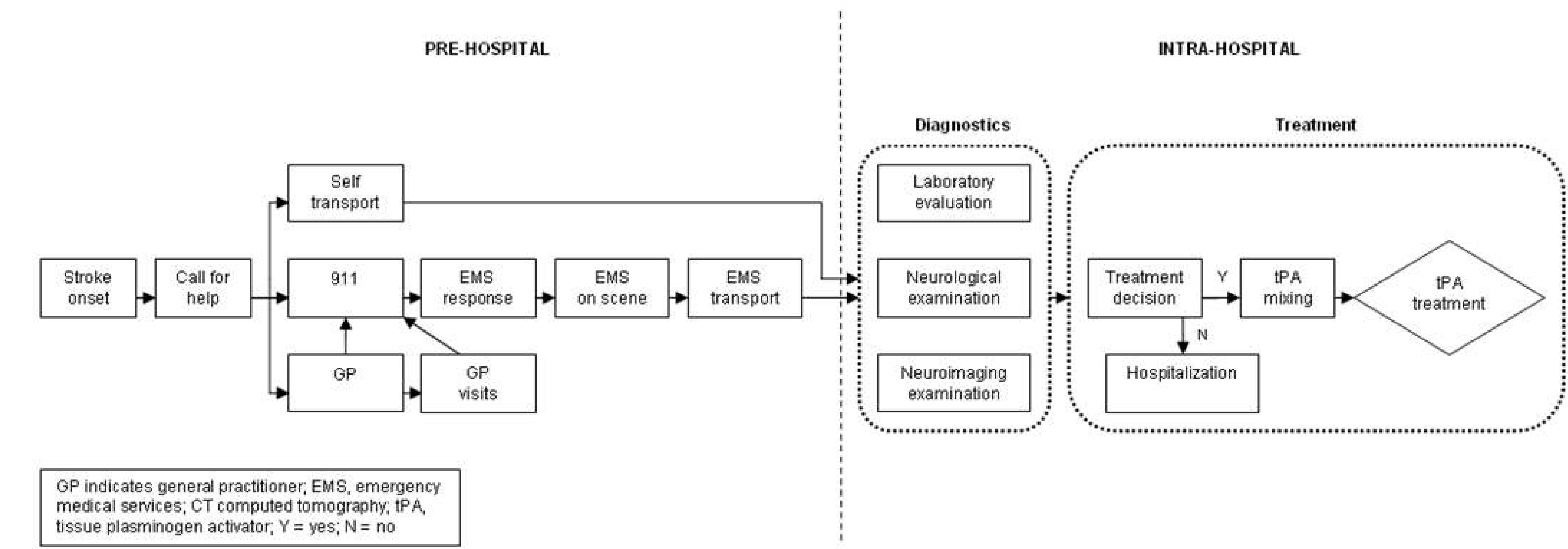

Table. Descriptive statistics of activity durations and diagnostics.

\begin{tabular}{lc}
\hline Number of patients & 280 \\
Age in years (SD) & $70(14)$ \\
Male (\%) & $156(56)$ \\
Patient responsiveness & \\
Time from symptom onset to call for help, valid cases (\%) & $152(54)$ \\
Median, minutes (IQR) & $41(5-130)$ \\
Mode of referal (\%) & \\
General practitioner & $135(48)$ \\
911 & $84(30)$ \\
Self-referral & $59(21)$ \\
Pathway set-up & \\
Transported by EMS ( $\%$ ) & $213(76)$ \\
Response time, median (min) & 9 \\
On scene time, median (min) & 20 \\
Transportation time, median (min) & 17 \\
Median time from hospital anival to neurological examination, min (IQR) & $2(0-0)$ \\
Median time from hospital anival to CT examination min (IQR) & $12(6-15)$ \\
Median time from hospital arival to laboratory examination, min (IQR) & $32(27-37)$ \\
Median door to IVT time, min (IQR) & $35(25-45)$ \\
\hline SD indicates standard deviation; IQR, Interquartile Range; EMS, Emergency Medical Services; CT, Computed \\
Tomography, IVT, Intavenous Thrombolysis.
\end{tabular}

Figure 2. Summary of best practices and relative gap between baseline model and optimized settings.

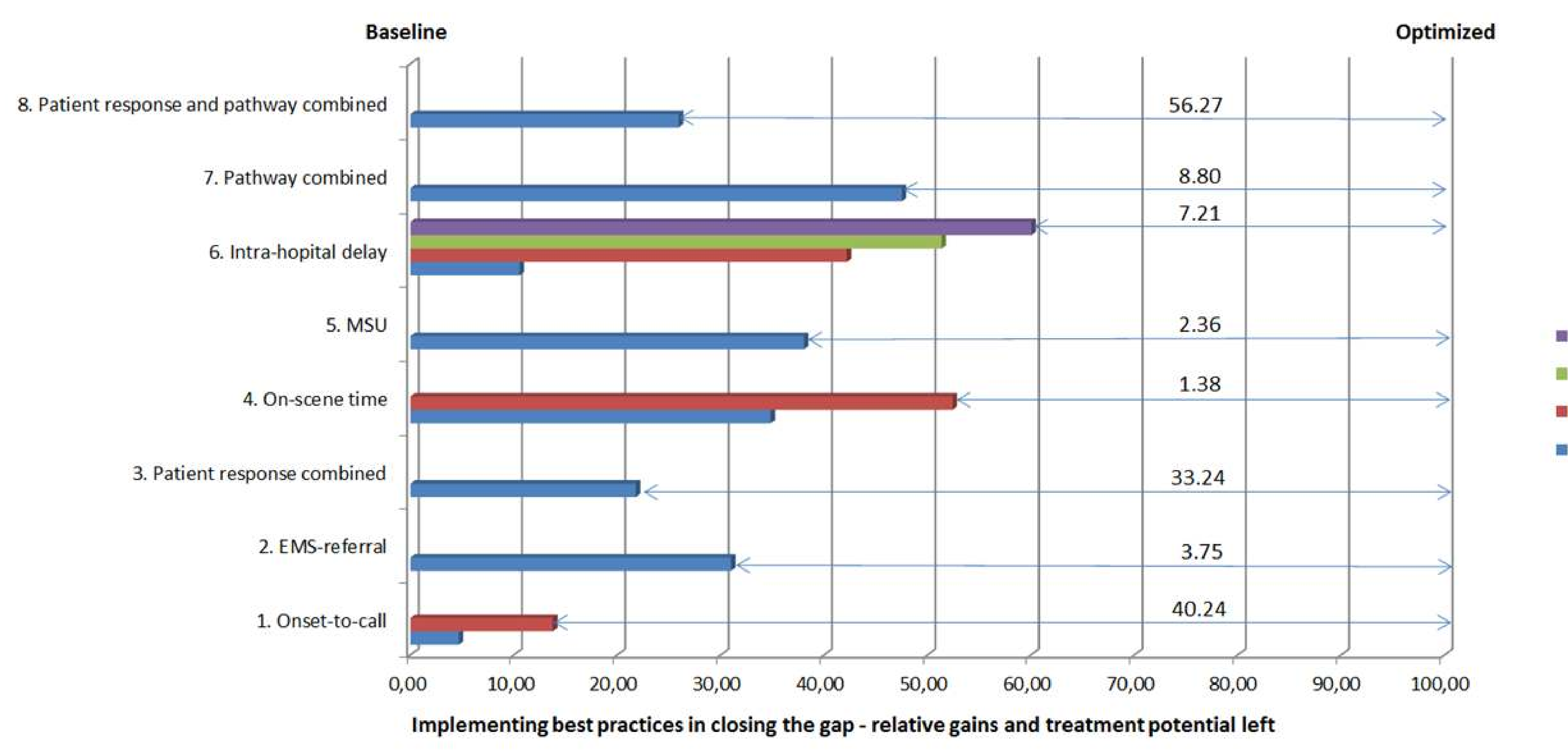

\section{DISCUSSION}

- We observed the greatest effects after reducing the time from symptom onset to the call for help, expediting intra-hospital care services and by increasing the number of 911 calls.

- Remaining obstacles might be difficult to overcome within the short term given the limited availability of solutions to further expedite care and logistical services at tolerable costs.

- The results and approach presented here may facilitate extension of the simulation model for the acute stroke pathway to include endovascular treatment.

\section{CONCLUSIONS}

- Results indicate that the cumulative effects of implementing best practices on the organization of stroke care would clearly exceed current benchmarks for treatment rates.

- A broader overview facilitated by simulation is suggested as instrumental in supporting decision-makers and clinicians in their efforts to evaluate the set-up and performance of acute stroke pathways.

1. Rose KM, Rosamond WD, Huston SL, Murphy CV, Tegeler CH. Predictors of time from hospital arrival to initial brain-imaging among suspected stroke patients: The north carolina collaborative stroke registry. Stroke 2008;39:3262-3267. 2. Meretoja A, Strbian D, Mustanoja S, Tatlisumak T, Lindsberg PJ, Kaste M. Reducing in-hospital delay to 20 minutes in stroke thrombolysis. Neurology 2012;79:306-13. 